\title{
Success of Face Analysis Technology in Rare Genetic Diseases Diagnosed by Whole-Exome Sequencing: A Single-Center Experience
}

\author{
Muhsin Elmas Basak Gogus \\ Department of Medical Genetics, Afyonkarahisar Health Sciences University, Afyonkarahisar, Turkey
}

\section{Keywords}

Artificial intelligence $\cdot$ Deep learning $\cdot$ Whole-exome sequencing

\begin{abstract}
The diagnosis of rare genetic diseases is one of the most difficult areas in medicine. Whole-exome sequencing (WES) technology makes it easier to diagnose these diseases. In addition, next-generation phenotyping can help to diagnose computer-based algorithms. Detailed dysmorphologic findings of 25 patients diagnosed by WES in our center were described. The success of this technology in diagnosing rare genetic diseases was investigated by scanning the photographs of 25 patients with Face2Gene application. The application listed possible preliminary diagnoses (30 disease suggestion). Of these, 12 (48\%) cases were correctly matched. The most common disease group in the patients was neurological disease (96\%). The most common mode of inheritance in the patients was autosomal recessive. The rate of consanguineous marriages was determined in $80 \%$ of the patients. Ten patients had microcephaly and 7 patients had corpus callosum anomaly. In our study, we found that the success of Face2Gene was lower than described in the literature. We think that the probable cause of this condition is that the cases are very rare, and there is not enough data about these
\end{abstract}

diseases in the application. Therefore, it is recommended that applications should be used more frequently by pediatricians and clinical geneticists. The diagnosis of rare diseases still is quite difficult. Nowadays, WES is a successful method. However, applications such as Face2Gene help to make a clinical prediagnosis and create a larger database.

(c) 2020 S. Karger AG, Basel

Diseases with genetic etiology affect most of the population during their lifetimes. Syndromic cases have symptoms that significantly reduce the quality of life of patients, and they affect about $8 \%$ of the population [Baird et al., 1988]. Genetic diagnosis is important in syndromic cases because, following a diagnosis, there are special prevention and screening programs available for primary and secondary symptoms.

Many of these syndromic cases have facial phenotypes and some characteristic facial features that provide a clue for the diagnosis of genetic diseases. Approximately 30 $40 \%$ of the genetic diseases show craniofacial alteration (as in Down syndrome, Fragile X, etc.) [Ferry et al., 2014].

Dysmorphology provides identification of before- or after-birth nonnormative forms that enables classification of various congenital malformations. This term provides a comparable description of all body characteristics

\section{KARGER}

(C) 2020 S. Karger AG, Basel

karger@karger.com

www.karger.com/msy
Muhsin Elmas, MD

Department of Medical Genetics, Afyonkarahisar Health Sciences University Zafer Sağlık Külliyesi, Dörtyol Mahallesi 2078, Sokak No. 3

Afyonkarahisar 03000 (Turkey)

E-Mail drmelmas@gmail.com 
(stature, feet, hands, neck) and face (such as shape of head, nose length, position of ears, thickness of vermillion, etc.) of the individuals of the same age group and ethnicity. A genetic etiology should be suspected if a child has a dysmorphic appearance with one of these features: (a) congenital anomalies, (b) growth retardation, (c) developmental delay and intellectual disability or developmental regression, (d) undeveloped secondary sexual characteristics, or (e) ambiguous genitalia [Featherstone et al., 2005; Smigiel and Demkow, 2016].

In the last 3 decades, with the advancement of technology, various databases have been created to determine the dysmorphic facial findings of patients (London Dysmorphology Database, Pictures Of Standard Syndromes and Undiagnosed Malformations Database, etc.) [Guest et al., 1999; Strømme, 1999]. These databases allow the comparison between the facial gestalt of patients and normal facial features, but these findings are affected by external factors such as available lighting conditions, posture of patients, etc. [Hammond, 2007]. In recent years, DeepGestalt program has been developed using computer technology and deep learning algorithms that measure similarities to hundreds of genetic syndromes based on unconstrained 2D images [Gurovich et al., 2018]. In a previous study, DeepGestalt achieved 91\% top-10 correctness in identifying the syndrome on 502 different patient photos [Gurovich et al., 2019]. The Face2Gene (FDNA Inc, Boston, USA) application is a novel framework based on DeepGestalt, and it is one of the next-generation phenotyping (NGP) technologies used to determine the phenotype in thousands of diseases and correlate this phenotype with the genotype. In addition to DeepGestalt, which is an analysis based on a frontal facial photo of the patient, Face2Gene also offers an analysis based on the clinical findings of the patient (feature match). In this study, we considered both types of analysis and included them.

With the development of technology, other methods have been developed for the diagnosis of genetic diseases using molecular genetic tests. One of these methods is whole-exome sequencing (WES). This technique allows sequencing of the whole protein-coding region of genes in a genome (known as the exome) [ $\mathrm{Ng}$ et al., 2009]. Exomes are about $2 \%$ of the whole genome, and mutations in these regions cause approximately $80 \%$ of mendelian diseases [Botstein and Risch, 2003; Yang et al., 2014]. In previous meta-analysis studies, the reported success rate of WES for molecular diagnosis was $24-68 \%$ (approximately 31\%) [Clark et al., 2018]. This method has become a good alternative for the molecular diagnosis of rare genetic diseases that have prevalence below $1 / 2,000$ [Ng et al., 2010].

Dysmorphic facial features are important to determine the causative variants of rare genetic diseases in WES. Therefore, when determining that pathogenic variants are causative of a disease or not, phenotypic features of patients are compared with similar previously reported cases using WES. For this reason, any type of clue (face gestalt, any prenatal findings, biochemical results, etc.) is increasingly important. In this study, we aimed to investigate the importance of determining dysmorphic facial features in rare genetic diseases with definitive molecular diagnosis using the WES method. We also evaluated the success of NGP technologies in this disease group.

\section{Materials and Methods}

The aim of this study was to determine the dysmorphic facial features of rare diseases diagnosed using WES and the power of NGP technology. This is a retrospective study. Therefore, the hospital registry system and patient files were reevaluated. The criteria for inclusion in the study are as follows: (1) patients were admitted to the Department of Medical Genetics at Afyonkarahisar University of Health Sciences between 2012-2019, (2) all patients had had sequencing results, (3) all patients had at least 1 front face image in the their files to evaluate the facial features, and (4) all patients had signed a consent form. Patients without adequate photographs to assess facial features were excluded from the study.

We selected 25 patients ( 9 females and 16 males) younger than 16 years of age who met all inclusion criteria. The photos and relevant clinical features were uploaded to Face2Gene, and the resulting analysis was assessed and correlated to the molecular diagnosis. We also checked how the correct diagnosis is ranked by both types of analysis, DeepGestalt and feature match. In addition, the research application of Face2Gene was used to understand whether the tool differentiated the group of patients from control groups [Pantel et al., 2018].

We used the hospital registry system to evaluate if the selected patients had anamnesis information, biochemical test results, MR images, echocardiographic results, and genetic reports. The parameters questioned in the anamnesis information were prenatal (oligohydroamnios, polyhydroamniosis, fetal akinesia, any abnormal ultrasound image), natal (delivery type, birth weight, gestation week at birth), postnatal stories (story of neonatal intensive care, hypoxic ischemic birth history, hypotonia, sucking difficulty), pedigree, presence of individuals with similar symptoms in the family, if consanguineous marriage (when yes, which degree), delay in intellectual/motor development stages, surgery history, and seizures.

Human Malformation Terminology (The Elements of Morphology) was used to determine the presence of any dysmorphic finding. This terminology was created by a group of clinicians working in the field of dysmorphology to standardize the definition of human morphology in which a reliable comparison of phenotypic findings among patients is provided [Allanson et al., 2009]. This terminology consists of 6 articles. These are standard termi- 
Table 1. Positive exome cases with pathogenic variants and secondary findings

\begin{tabular}{|c|c|c|c|c|c|c|c|c|}
\hline $\begin{array}{l}\text { Case } \\
\text { ID }\end{array}$ & Genes & OMIM & $\begin{array}{l}\text { Mode of } \\
\text { inheritance }\end{array}$ & $\begin{array}{l}\text { Consanguineous } \\
\text { marriage }\end{array}$ & $\begin{array}{l}\text { Other affected } \\
\text { individuals }\end{array}$ & Mutations & Zygosity & Genetic diagnosis \\
\hline P1 & COL6A2 & 120240 & $\begin{array}{l}\text { Autosomal } \\
\text { recessive }\end{array}$ & Same village & No & c. $2584 \mathrm{C}>\mathrm{T}$ (p.Arg862Trp) (p.R862W) & Homozygous & Bethlem myopathy 1 \\
\hline P2 & COG5 & 613612 & $\begin{array}{l}\text { Autosomal } \\
\text { recessive }\end{array}$ & $\begin{array}{l}\text { First-degree } \\
\text { cousin marriage }\end{array}$ & 1 male sibling & hg19:chr7:107002545A>G; c.1052T>C; p.Leu351Pro & Homozygous & $\begin{array}{l}\text { Congenital disorder of } \\
\text { glycosylation, type IIi }\end{array}$ \\
\hline P3 & SPG11 & 604360 & $\begin{array}{l}\text { Autosomal } \\
\text { recessive }\end{array}$ & $\begin{array}{l}\text { First-degree } \\
\text { cousin marriage }\end{array}$ & No & hg19:44925739; c.1699C>T; p.Gln567* & Homozygous & $\begin{array}{l}\text { Spastic paraplegia } 11, \\
\text { autosomal recessive }\end{array}$ \\
\hline P4 & POMT1 & 607423 & $\begin{array}{l}\text { Autosomal } \\
\text { recessive }\end{array}$ & $\begin{array}{l}\text { Second-degree } \\
\text { cousin marriage }\end{array}$ & No & c.598G >C (p.Ala200Pro) (p.A200P) & Homozygous & $\begin{array}{l}\text { Muscular dystrophydystrogly- } \\
\text { canopathy (congenital with } \\
\text { mental retardation), type B, } 1\end{array}$ \\
\hline P5 & $L A R P 7$ & 612026 & $\begin{array}{l}\text { Autosomal } \\
\text { recessive }\end{array}$ & $\begin{array}{l}\text { First-degree } \\
\text { cousin marriage }\end{array}$ & $\begin{array}{l}1 \text { female sibling } \\
\text { (case 6) }\end{array}$ & $\begin{array}{l}\text { NM_015454.1_c.802_1142+267del } \\
\left(\text { p.Lys } 278^{\star} / \text { c.832A }>\text { T) }\right.\end{array}$ & Homozygous & Alazami syndrome \\
\hline P6 & $L A R P 7$ & 612026 & $\begin{array}{l}\text { Autosomal } \\
\text { recessive }\end{array}$ & $\begin{array}{l}\text { First-degree } \\
\text { cousin marriage }\end{array}$ & $\begin{array}{l}1 \text { male sibling } \\
\text { (case 5) }\end{array}$ & $\begin{array}{l}\text { NM_015454.1_c.802_1142+267del } \\
\text { (p.Lys } 278^{\star} / \text { c.832A }>\text { T) }\end{array}$ & Homozygous & Alazami syndrome \\
\hline P7 & THOC6 & 615403 & $\begin{array}{l}\text { Autosomal } \\
\text { recessive }\end{array}$ & $\begin{array}{l}\text { First-degree } \\
\text { cousin marriage }\end{array}$ & $\begin{array}{l}1 \text { male sibling } \\
\text { (case } 8 \text { ) }\end{array}$ & Exon11: c.709delG; p.G237fs; chr16:3077179_AG>A & Homozygous & $\begin{array}{l}\text { Beaulieu-Boycott-Innes } \\
\text { syndrome }\end{array}$ \\
\hline P8 & THOC6 & 615403 & $\begin{array}{l}\text { Autosomal } \\
\text { recessive }\end{array}$ & $\begin{array}{l}\text { First-degree } \\
\text { cousin marriage }\end{array}$ & $\begin{array}{l}1 \text { male sibling } \\
\text { (case 7) }\end{array}$ & Exon11: c.709delG; p.G237fs; chr16:3077179_AG>A & Homozygous & $\begin{array}{l}\text { Beaulieu-Boycott-Innes } \\
\text { syndrome }\end{array}$ \\
\hline P9 & $N A G L U$ & 609701 & $\begin{array}{l}\text { Autosomal } \\
\text { recessive }\end{array}$ & $\begin{array}{l}\text { First-degree } \\
\text { cousin marriage }\end{array}$ & No & c.1694G>A (p.Arg565Gln) (p.R565Q) & Homozygous & $\begin{array}{l}\text { Mucopolysaccharidosis type } \\
\text { IIIB (Sanfilippo B) }\end{array}$ \\
\hline $\mathrm{P} 10$ & KLHL7 & 611119 & $\begin{array}{l}\text { Autosomal } \\
\text { recessive }\end{array}$ & $\begin{array}{l}\text { Third-degree } \\
\text { cousin marriage }\end{array}$ & $\begin{array}{l}1 \text { male sibling } \\
\text { (case 11) }\end{array}$ & Exon 8: c.C1051T; p.R351X & Homozygous & $\begin{array}{l}\text { Crisponi/CISS1-like phenotype } \\
\text { associated with early-onset } \\
\text { retinitis pigmentosa }\end{array}$ \\
\hline P11 & KLHL7 & 611119 & $\begin{array}{l}\text { Autosomal } \\
\text { recessive }\end{array}$ & $\begin{array}{l}\text { Third-degree } \\
\text { cousin marriage }\end{array}$ & $\begin{array}{l}1 \text { male sibling } \\
\text { (case 10) }\end{array}$ & Exon 8: c.C1051T; p.R351X & Homozygous & $\begin{array}{l}\text { Crisponi/CISS1-like phenotype } \\
\text { associated with early-onset } \\
\text { retinitis pigmentosa }\end{array}$ \\
\hline P12 & $V L D L R$ & 192977 & $\begin{array}{l}\text { Autosomal } \\
\text { recessive }\end{array}$ & $\begin{array}{l}\text { Second-degree } \\
\text { cousin marriage }\end{array}$ & $\begin{array}{l}1 \text { male sibling } \\
\text { (case 13) }\end{array}$ & NM_001018056.1:c.1459G>T; p.Asp487Tyr & Homozygous & $\begin{array}{l}\text { Cerebellar hypoplasia and } \\
\text { mental retardation with or } \\
\text { without quadrupedal } \\
\text { locomotion } 1\end{array}$ \\
\hline P13 & $V L D L R$ & 192977 & $\begin{array}{l}\text { Autosomal } \\
\text { recessive }\end{array}$ & $\begin{array}{l}\text { Second-degree } \\
\text { cousin marriage }\end{array}$ & $\begin{array}{l}1 \text { male sibling } \\
\text { (case 12) }\end{array}$ & NM_001018056.1:c.1459G>; p.Asp487Tyr/ & Homozygous & $\begin{array}{l}\text { Cerebellar hypoplasia and } \\
\text { mental retardation with or } \\
\text { without quadrupedal } \\
\text { locomotion } 1\end{array}$ \\
\hline P14 & $T B C D$ & 604649 & $\begin{array}{l}\text { Autosomal } \\
\text { recessive }\end{array}$ & Same village & No & c.230A > G (p.His77Arg) (p.H77R) & Homozygous & $\begin{array}{l}\text { Encephalopathy, progressive, } \\
\text { early-onset, with brain atrophy } \\
\text { and thin corpus callosum }\end{array}$ \\
\hline P15 & COG6 & 606977 & $\begin{array}{l}\text { Autosomal } \\
\text { recessive }\end{array}$ & $\begin{array}{l}\text { First-degree } \\
\text { cousin marriage }\end{array}$ & 1 female cousin & Exon8: c.G697A; p.Glu233Lys & Homozygous & $\begin{array}{l}\text { Congenital disorder of } \\
\text { glycosylation, type IIl }\end{array}$ \\
\hline P16 & COG6 & 606977 & $\begin{array}{l}\text { Autosomal } \\
\text { recessive }\end{array}$ & $\begin{array}{l}\text { First-degree } \\
\text { cousin marriage }\end{array}$ & 1 female cousin & Exon8: c.G697A; p.Glu233Lys & Homozygous & $\begin{array}{l}\text { Congenital disorder of } \\
\text { glycosylation, type IIl }\end{array}$ \\
\hline P17 & LAMA1 & 615960 & $\begin{array}{l}\text { Autosomal } \\
\text { recessive }\end{array}$ & Same village & $\begin{array}{l}1 \text { male sibling } \\
\text { (case } 18 \text { ) }\end{array}$ & c.8192C $>$ A (p.S2731*) (p.Ser2731Ter) & Homozygous & $\begin{array}{l}\text { Poretti-Boltshauser Syndrome; } \\
\text { PTBHS }\end{array}$ \\
\hline $\mathrm{P} 18$ & LAMA1 & 615960 & $\begin{array}{l}\text { Autosomal } \\
\text { recessive }\end{array}$ & Same village & $\begin{array}{l}1 \text { male sibling } \\
\text { (case 17) }\end{array}$ & c.8192C $>$ A (p.S2731*) (p.Ser2731Ter) & Homozygous & $\begin{array}{l}\text { Poretti-Boltshauser Syndrome; } \\
\text { PTBHS }\end{array}$ \\
\hline P19 & $L A R P 7$ & 612026 & $\begin{array}{l}\text { Autosomal } \\
\text { recessive }\end{array}$ & $\begin{array}{l}\text { Second-degree } \\
\text { cousin marriage }\end{array}$ & 2 male cousins & NM_001267039.1(LARP7):c.441del & Homozygous & Alazami syndrome \\
\hline P20 & $T P P 1$ & 607998 & $\begin{array}{l}\text { Autosomal } \\
\text { recessive }\end{array}$ & $\begin{array}{l}\text { Second-degree } \\
\text { cousin marriage }\end{array}$ & No & hg19:chr11:6639009T>C; c.230-2A>G;p.? & Homozygous & $\begin{array}{l}\text { Ceroid Lipofuscinosis, } \\
\text { Neuronal, 2; CLN2 }\end{array}$ \\
\hline P21 & MASP1 & 600521 & $\begin{array}{l}\text { Autosomal } \\
\text { recessive }\end{array}$ & $\begin{array}{l}\text { First-degree } \\
\text { cousin marriage }\end{array}$ & No & $\begin{array}{l}\text { hg19:chr3:186954244G>A; c.1415C>T; p.Thr472Ile } \\
\text { hg19:chr3:186980508C>A; c.238G>T; p.Val80Leu }\end{array}$ & $\begin{array}{l}\text { Compound } \\
\text { heterozygous }\end{array}$ & $3 \mathrm{MC}$ syndrome 1 \\
\hline P22 & GPT2 & 138210 & $\begin{array}{l}\text { Autosomal } \\
\text { recessive }\end{array}$ & $\begin{array}{l}\text { Second-degree } \\
\text { cousin marriage }\end{array}$ & No & hg19:chr16:46960903G>A; c.1435G>A; p.Val479Met & Homozygous & $\begin{array}{l}\text { Intellectual disability, } \\
\text { autosomal recessive } 49\end{array}$ \\
\hline P23 & OCLN & 602876 & $\begin{array}{l}\text { Autosomal } \\
\text { recessive }\end{array}$ & $\begin{array}{l}\text { First-degree } \\
\text { cousin marriage }\end{array}$ & $\begin{array}{l}1 \text { male sibling } \\
\text { (case 24) }\end{array}$ & hg19:chr5:68830667G >A; c. $1037+1 \mathrm{G}>\mathrm{A}$ & Homozygous & Pseudo-TORCH syndrome 1 \\
\hline P24 & OCLN & 602876 & $\begin{array}{l}\text { Autosomal } \\
\text { recessive }\end{array}$ & $\begin{array}{l}\text { First-degree } \\
\text { cousin marriage }\end{array}$ & $\begin{array}{l}1 \text { male sibling } \\
\text { (case 23) }\end{array}$ & hg19:chr5:68830667G >A; c. $1037+1 \mathrm{G}>\mathrm{A}$ & Homozygous & Pseudo-TORCH syndrome 1 \\
\hline P25 & ACSL4 & 300157 & $\begin{array}{l}\text { X-linked } \\
\text { dominant }\end{array}$ & Same village & Mother & hg19:chrX:108926022G>T; c.455C>A; p.Thr152Asn & Hemizygous & $\begin{array}{l}\text { Intellectual disability, X-linked } \\
63\end{array}$ \\
\hline
\end{tabular}


nology for the head and face; the periorbital region; the ear; the nose and philtrum; the lips, mouth, and oral region as well as the hands and feet.

WES analysis of the patients was performed by the contracted institutions or the universities we worked with in this study, and the test results were examined extensively. If the same gene was found to cause 2 or more diseases, differential diagnosis was made by detailed physical examination. Dysmorphic findings had a very important role at this stage. MR images and echocardiography findings were also used.

WES analysis findings of patients, anamnesis information, pedigree analysis, and clinical findings were collected. Dysmorphic facial features were evaluated in detail. Since dysmorphic facial features are a diagnostic clue to medical genetic doctors, we aimed to present dysmorphic facial findings of patients diagnosed as rare genetic diseases in our clinic. In this study, we evaluated the power of Face2Gene, a new NGP technology that makes recommendations for possible genetic disease using face gestalt information. Therefore, when choosing which test to perform in the diagnosis of rare genetic disease, we also evaluated the necessity of the recommendations of this next-generation approach.

\section{Results}

The disease-causing mutations of all patients and their demographics are presented in Table 1. According to the mutation points, physical examination findings, laboratory and imaging tests, results were reevaluated and a definitive diagnosis was determined. Except for 1 patient, all of the diagnosed diseases were of autosomal recessive inheritance. In terms of consanguinity, consanguineous marriages were found in $80 \%$ (12 of them were first-degree cousins, 6 of them were second-degree, and 2 of them were third-degree cousins). No consanguinity was defined for parents of the other 5 patients, but they were from the same village (except for the parents of P25).

The symptoms and dysmorphic facial features in our patients are summarized in Table 2. Almost all of the patients were evaluated as neurological diseases (only 1 patient had a storage disorder). All other patients had neuromotor growth retardation and different degrees of learning disability. Ten patients (40\%) had microcephaly. Two patients had muscle weakness, and 3 patients had persistent hypotonia. Only 1 patient had inadequacy in cerebellar tests. Twenty-two of the 25 patients had an MRI. When MRI findings were examined, 4 patients had normal findings, and 18 patients had abnormal findings. The most common findings were corpus callosum anomalies (7/18). Other anomalies were periventricular hyperintensity (4/18), dilated ventricles (4/18), and cortical atrophy (3/18), respectively.

Whole-Exome Sequencing and Artificial Intelligence
Clinical findings of all patients were annotated in Face2Gene application. The most common clinical finding was global developmental delay (16\%). The second most common findings were inability to walk, delayed speech and language development, hypospadias, hypoplasia of corpus callosum, and short stature (12\% for all). The third most common findings were hypothyroidism, hyperreflexia and facial hypertrichosis ( $8 \%$ for all), and the fourth most common findings were hyperactive patellar reflex, hydrocephalus, hepatomegaly, postnatal growth retardation, equinovarus deformity and epiphyseal dysplasia ( $4 \%$ for all). The distribution of clinical features is shown in Figure 1.

Among the 30 diseases, the syndromes recommended by Face2Gene were analyzed for the presence and sequence of the syndrome diagnosed. Twelve of the cases (48\%) had a correct match. The remaining 13 cases had a diagnosis that was not part of the 300 syndromes that DeepGestalt currently identifies. Thus, we only relied on the feature match algorithm. The application ranks the suggested syndromes based on 2 scores: Gestalt score and Feature score. Gestalt score is a value obtained by analyzing the photograph of the patient (dysmorphic facial features). The Feature score is a value obtained by analyzing the annotated clinical findings [Gurovich et al., 2019]. Gestalt score was calculated for only one (P9) of the patients included in this study. The other 11 patients had a Gestalt score as 0 . Therefore, the Feature score is important for these patients. This situation shows the importance of the entry of the clinical feature findings of the patients to the application.

\section{Discussion}

In this study, we presented the importance of dysmorphic findings in rare genetic diseases and the power of the DeepGestalt program, which enables facial analysis for genetic syndrome classification. This application helps clinicians to make a definitive diagnosis of the disease. With further development of this application, it may be possible to use it routinely in genetic polyclinics.

Prevalence of rare genetic diseases is between 6 and $8 \%$ [Lodato and Kaplan, 2013]. In this group of diseases, any clinical (prenatal, natal, and postnatal) and dysmorphic findings are of importance at the stage of prediagnosis. In this study, we presented the clinical, radiological, and dysmorphic features of patients with rare genetic diseases diagnosed by WES analyses. Regarding that clinical geneticists and pediatricians rarely encounter this group of 
Table 2. Summary of clinical and dysmorphic findings of patients

\begin{tabular}{|c|c|c|c|}
\hline Case ID & Presenting symptoms & Dysmorphic facial findings & MRI findings \\
\hline $\mathrm{P} 1$ & $\begin{array}{l}\text { Decreased fetal movements at intrauterine } \\
\text { period, resistant epilepsy, neuromotor } \\
\text { developmental delay, severe learning } \\
\text { disability, microcephaly, upper and lower } \\
\text { limb muscle weakness, pes equinusvarus, } \\
\text { spasticity, contracture lower extremities, } \\
\text { increased deep tendon reflexes, proximal } \\
\text { muscle atrophy, strabismus }\end{array}$ & $\begin{array}{l}\text { Brachycephaly, flat occiput, square face, narrow forehead, sloping forehead, } \\
\text { malar flatting, pointed chin, deeply set eyes, thick eyebrows, almond-shaped } \\
\text { palpebral fissure, telecanthus, prominent inferior crus of antihelix, } \\
\text { prominent antihelix stem, prominent superior crus of antihelix, } \\
\text { underdeveloped crus helix, large lobe, bifid tragus, low insertion of } \\
\text { columella, enlarged nares, wide nasal bridge, depressed nasal bridge, wide } \\
\text { nasal ridge, midline sinus of philtrum, thin upper lip vermilion, thick lower } \\
\text { lip vermilion }\end{array}$ & $\begin{array}{l}\text { Hydro- } \\
\text { cephalus, } \\
\text { thin corpus } \\
\text { callosum }\end{array}$ \\
\hline $\mathrm{P} 2$ & $\begin{array}{l}\text { Neuromotor developmental delay, mild } \\
\text { learning disability, dysmetria and } \\
\text { dysdiadochokinesia, seizure, imbalance to } \\
\text { especially right side, generalized } \\
\text { hypertrichosis, dysmorphic facial features }\end{array}$ & $\begin{array}{l}\text { Square face, broad forehead, premaxillary underdevelopment, broad jaw, full } \\
\text { cheeks, broad and horizontal eyebrow, long palpebral fissure, synophrys, } \\
\text { telecanthus, long ear, underdeveloped crus helix, underdeveloped tragus, } \\
\text { broad columella, wide nasal bridge, broad philtrum, smooth philtrum, } \\
\text { prominent nasolabial fold, thick low and upper lip vermilion }\end{array}$ & $\begin{array}{l}\text { Diffuse } \\
\text { cerebellar } \\
\text { atrophy }\end{array}$ \\
\hline P3 & $\begin{array}{l}\text { Walking disability, moderate learning } \\
\text { disability, decrease of muscular power at } \\
\text { extremities, bilateral } 5 \text { th trigger finger, foot } \\
\text { spasticity, increase of deep tendon reflex, } \\
\text { pes cavus }\end{array}$ & $\begin{array}{l}\text { Hypertelorism, horizontal eyebrow, downslanted palpebral fissure, } \\
\text { telecanthus, prominent antitragus, expanded terminal portion of crus helix, } \\
\text { macrotia, broad columella, low insetion of columella, enlarged nares, wide } \\
\text { nasal bridge, wide nasal ridge, broad nasal tip, thin upper lip vermilion, } \\
\text { diastema in anterior incisor teeth }\end{array}$ & $\begin{array}{l}\text { Periventricular } \\
\text { hyperintensity }\end{array}$ \\
\hline $\mathrm{P} 4$ & $\begin{array}{l}\text { Global developmental delay, severe learning } \\
\text { disability, skeletal muscle weakness, } \\
\text { increased creatinine level, macroglossia, } \\
\text { joint contractures, spinal scoliosis, } \\
\text { hypertrichosis }\end{array}$ & $\begin{array}{l}\text { Brachycephaly, flat occiput, triangular face, narrow forehead, prominent } \\
\text { cheekbone, prominent nasolabial fold, broad chin, long palpebral fissure, } \\
\text { prominent antihelix stem, prominent antitragus, expanded terminal portion } \\
\text { of crus helix, wide nasal base, wide nasal bridge, broad nasal tip, deep } \\
\text { philtrum, everted lower lip vermilion, thin lower lip vermilion, downturned } \\
\text { corners of mouth, wide mouth }\end{array}$ & Normal \\
\hline P5 & $\begin{array}{l}\text { Neuromotor developmental delay, severe } \\
\text { learning disability, microcephaly, autistic } \\
\text { behaviors, pulmonary stenosis, epiphyseal } \\
\text { changes in the proximal phalanges, } \\
\text { strabismus, tortuous at retinal blood vessels, } \\
\text { hyperkeratosis, diabetes mellitus type } 2 \text {, } \\
\text { short stature, pes planus }\end{array}$ & $\begin{array}{l}\text { Brachycephaly, coarse face, malar prominence, midface prominence, } \\
\text { prominent nasolabial fold, broad chin, hypertelorism, deep-set eyes, broad } \\
\text { eyebrow, infraorbital crease, angulated antihelix, underdeveloped antihelix } \\
\text { crus superior, prominent antitragus, long ear, low-set ears, protruding ears, } \\
\text { anterior crease lobes, large lobes, macrotia, broad columella, large nose, wide } \\
\text { nasal base, depressed nasal bridge, wide nasal bridge, wide nasal ridge, broad } \\
\text { nasal tip, long nose, prominent nose, short philtrum, tented philtrum, wide } \\
\text { mouth, macrostomia, large tongue }\end{array}$ & $\begin{array}{l}\text { Arnold Chiari } \\
\text { malformation } \\
\text { type } 1\end{array}$ \\
\hline P6 & $\begin{array}{l}\text { Neuromotor developmental delay, severe } \\
\text { learning disability, seizure, microcephaly, } \\
\text { autistic behaviors, pulmonary stenosis, } \\
\text { hyperkeratosis }\end{array}$ & $\begin{array}{l}\text { Brachycephaly, short face, square face, full cheeks, malar prominence, } \\
\text { midface prominence, premaxillary prominence, broad chin, short chin, } \\
\text { hypertelorism, laterally extended eyebrow, deep-set eyes, infraorbital fold, } \\
\text { telecanthus, long ears, protruding ears, large lobe, broad columella, large } \\
\text { nose, wide nasal base, depressed nasal bridge, wide nasal bridge, wide nasal } \\
\text { ridge, broad nasal tip, long nose, short philtrum, tented philtrum, wide } \\
\text { mouth, thick lower lip vermilion, macrostomia }\end{array}$ & $\begin{array}{l}\text { Corpus } \\
\text { callosum } \\
\text { agenesis, } \\
\text { distinct } \\
\text { vascular } \\
\text { structures, } \\
\text { venous } \\
\text { angioma }\end{array}$ \\
\hline P7 & $\begin{array}{l}\text { Global developmental delay, severe learning } \\
\text { disability, autistic behaviors, microcephaly, } \\
\text { early closure of anterior fontanelle, lack of } \\
\text { eye contact, criptorchidism, left } \\
\text { equinovarus foot deformity }\end{array}$ & $\begin{array}{l}\text { Brachycephaly, long face, underdeveloped supraorbital ridges, } \\
\text { underdevelopment cheekbone, malar flattening, underdeveloped nasolabial } \\
\text { fold, premaxillary, prominence, narrow jaw, pointed chin, hypertelorism, } \\
\text { almond-shaped palpebral fissure, telecanthus, prominent antihelix stem, } \\
\text { underdeveloped crus superior antihelix, long ears, small lobe, broad } \\
\text { columella, anteverted nares, wide nasal base, prominent nasal bridge, wide } \\
\text { nasal bridge, wide nasal ridge, broad nasal tip, bulbouse nose, smooth } \\
\text { philtrum, absent Cupid's bow, everted lower lip vermilion, thick lower lip } \\
\text { vermilion }\end{array}$ & Normal \\
\hline P8 & $\begin{array}{l}\text { Neuromotor developmental delay, severe } \\
\text { learning disability, autistic behaviors, } \\
\text { microcephaly, seizure, lack of eye contact, } \\
\text { criptorchidism }\end{array}$ & $\begin{array}{l}\text { Brachycephaly, flat occiput, full checks, premaxillary prominence, pointed } \\
\text { chin, hypertelorism, almond-shaped palpebral fissure, upslanted palpebral } \\
\text { fissure, telecanthus, prominent antihelix stem, prominent antihelix inferior } \\
\text { crus, prominent antihelix superior crus, angulated antihelix, everted } \\
\text { antitragus, protruding ear, crus helix connected to antihelix, small lobe, long } \\
\text { ears, low-hanging columella, wide nasal base, depressed nasal bridge, wide } \\
\text { nasal ridge, bulbouse nose, short philtrum, everted upper lip vermilion, thick } \\
\text { upper lip vermilion }\end{array}$ & Hydrocephalus \\
\hline
\end{tabular}


Table 2 (continued)

\begin{tabular}{lll}
\hline Case ID & Presenting symptoms & Dysmorphic facial findings \\
\hline P9 & $\begin{array}{l}\text { Speech disability, developmental delay, } \\
\text { hepatomegaly, mild learning disability, } \\
\text { ptosis left eye, otitis media with effusion }\end{array}$ & $\begin{array}{l}\text { Square face, malar flatting, underdeveloped nasolabial fold, broad jaw, broad } \\
\text { chin, epicanthus, hypertelorism, downslanted palpebral fissure, telecanthus, } \\
\text { prominent anithelix stem, serpenginous antihelix stem, everted antitragus, } \\
\end{array}$ \\
& $\begin{array}{l}\text { low-set ear, protruding ear, prominent crus helix, uplifted lobe, short } \\
\text { columella, anteverted nares, wide nasal bridge, thick lower lip vermilion }\end{array}$
\end{tabular}
columella, anteverted nares, wide nasal bridge, thick lower lip vermilion

P10 Neuromotor developmental delay, inability to walk, inability to talk, spasticity, microcephaly, severe learning disability, fetal akinesia, intrauterine growth restriction, perinatal asphyxia, Y-shaped syndactyly, hypospadias, scoliosis, intestinal obstruction

P11 Neuromotor developmental delay, inability to walk, inability to talk, spasticity, microcephaly, severe learning disability, fetal akinesia, perinatal asphyxia, Y-shaped syndactyly, hypospadias, scoliosis, intestinal obstruction, abnormal EEG

P12 Neuromotor developmental delay, moderate learning disability, delayed psychosocial development, macrocephaly, chronic constipation, strabismus
Narrow forehead, cheekbone underdevelopment, full cheeks, malar flatting, underdeveloped nasolabial fold, tall chin, hypertelorism, thick eyebrows, long eyelashes, prominent eyelashes, upslanted palpebral fissure, telecanthus, underdeveloped antitragus, low-set ears, expanded terminal portion of crus helix, underfolded helix, attached lobe, underdeveloped tragus, underdeveloped ala nasi, short columella, anteverted nares, depressed nasal bridge, wide nasal bridge, wide nasal ridge, depressed nasal tip, broad philtrum, deep philtrum, exaggerated Cupid's bow, thick lower lip vermilion

Frontal balding, narrow forehead, prominent forehead, underdeveloped supraorbital ridges, full cheeks, malar flatting, prominent nasolabial fold, premaxillary prominence, pointed chin, hypertelorism, thick eyebrow, long eyelashes, infra-orbital crease, telecanthus, antihelical shelf, underdeveloped antitragus, cupped ear, low-set ears, protruding ears, expanded terminal portion of crus helix, prominent crus helix, underfolded helix, small lobes, broad columella, low-hanging columella, wide nasal base, nasal bridge depressed, wide nasal bridge, convex nasal ridge, wide nasal ridge, broad nasal tip, midline sinus of philtrum, smooth philtrum, thick lower lip vermilion, thin upper lip vermilion

High anterior hairline, long face, narrow forehead, narrow jaw, pointed chin, tall chin, hypertelorism, sparse eyebrow, long palpebral fissure, telecanthus, prominent inferior crus of antihelix, prominent antihelix stem, prominent superior crus of antihelix, everted antitragus, expanded terminal portion of crus helix, prominent crus helix, low insertion of columella, prominent nasal bridge, broad nasal tip, bulbouse nose, long nose, short philtrum, smooth philtrum, wide mouth, microdontia

Prominent forehead, full cheeks, premaxillary prominence, short chin, deeply set eye, laterally extended eyebrow, sparse eyebrow, long eyelashes, proptosis, telecanthus, prominent inferior crus of antihelix, prominent antihelix stem, prominent antitragus, low-set ears, expanded terminal portion of crus helix, prominent crus helix, localized underdeveloped helix, bifid tragus, broad columella, depressed nasal bridge, wide nasal bridge, broad nasal tip, bulbouse nasal tip, broad philtrum

Brachycephaly, flat occiput, flat face, prominent glabella, malar flatting, pointed chin, epicanthus inversus, hypertelorism, deeply set eye, sparse eyebrow, prominent eyelashes, serpenginous antihelix stem, underdeveloped antitragus, cupped ears, low-set ears, expanded terminal portion of crus helix, prominent crus helix, overfolded helix, forward facing lobe, underdeveloped tragus, low-hanging columella, depressed nasal bridge, nasal bridge narrow, midline sinus philtrum, smooth philtrum, U-shaped upper lip vermilion
MRI findings

Thin corpus callosum, mucosal thickening at the paranasal sinuses, hyperintense cells in left mastoid cells

Periventricular hyperintensity, thin corpus callosum, dilated third and lateral ventricles

Dilated lateral ventricles, plagiocephaly

Dilated at 4 th ventricles, vermis and bilateral cerebellar hemisphere hypoplasia, pachygyria

Periventricular hyperintensity, vermis and bilateral cerebellar hemisphere hypoplasia, pachygyria

Bifrontotemporal atrophy, dilated bilateral ventricles and 3rd ventricular, periventricular hyperintensity, thin corpus callosum
Whole-Exome Sequencing and Artificial Intelligence
Mol Syndromol 2020;11:4-14

DOI: $10.1159 / 000505800$ 
Table 2 (continued)

\begin{tabular}{lll}
\hline Case ID & Presenting symptoms & Dysmorphic facial findings \\
\hline P15 & $\begin{array}{l}\text { Mental motor retardation, seizure, patent } \\
\text { ductus arteriosus, cryptorchidism, } \\
\text { hypothyroidism }\end{array}$ & $\begin{array}{l}\text { Flat occiput, round face, midface prominence, broad chin, long chin, } \\
\text { epicanthus, deep-set eyes, infraorbital crease, long palpebral fissure, } \\
\text { synophrys, telecanthus, prominent antihelix stem, prominent antitragus, } \\
\text { low-set ears, expanded terminal portion of crus helix, broad columella, wide } \\
\text { nasal base, depressed nasal bridge, wide nasal bridge, wide nasal ridge, } \\
\text { concave nasal ridge, broad nasal tip, long nose, broad philtrum, short } \\
\text { philtrum, smooth philtrum, absent Cupid's bow, U-shaped upper lip } \\
\text { vermilion }\end{array}$ \\
\hline $\begin{array}{l}\text { Mental motor retardation, intrauterine } \\
\text { growth restriction, perinatal asphyxia, } \\
\text { seizure, patent ductus arteriosus, bilateral } \\
\text { iris coloboma, hypothyroidism, horizontal } \\
\text { nystagmus }\end{array}$ & $\begin{array}{l}\text { Flat occiput, midface prominent, broad chin, bilateral blepharophimosis, } \\
\text { synophrys, telecanthus, prominent antihelix stem, prominent antitragus, } \\
\text { low-set ears, expanded terminal portion of crus helix, small lobe, wide nasal } \\
\text { base, depressed nasal bridge, wide nasal bridge, wide nasal ridge, broad nasal } \\
\text { tip, long nose, broad philtrum, short philtrum, smooth philtrum, absent }\end{array}$ \\
& Cupid's bow, thick lower lip vermilion, U-shaped upper lip vermilion
\end{tabular}

P17 Motor development delay, intrauterine growth restriction, cerebellar ataxia, mild learning disability
Flat face, midface prominence, underdeveloped nasolabial fold, pointed chin, telecanthus, prominent antihelix stem, low-set ears, unfolded helix, short columella, wide nasal base, wide nasal ridge, smooth philtrum, thin lower lip vermilion, thin upper lip vermilion
Multiple cysts in millimetric dimensions at both cerebellar hemispheres, irregularities in the cerebellum cortex, cerebellar vermis hypoplasia and enlarged at 4 th ventricle

\begin{tabular}{|c|c|c|c|}
\hline $\mathrm{P} 18$ & $\begin{array}{l}\text { Motor development delay, intrauterine } \\
\text { growth restriction, mirocephaly, } \\
\text { encephalocele, spasticity at lower and upper } \\
\text { extremities, blindness, cerebellar ataxia, } \\
\text { mild learning disability, seen lateral } \\
\text { ventricles and 3rd ventricular collapsed at } \\
\text { CT }\end{array}$ & $\begin{array}{l}\text { Brachycephaly, flat occiput, flat face, underdeveloped nasolabial fold, } \\
\text { upslanted palpebral fissures, telecanthus, low-set ears, protruding ears, thick } \\
\text { ala nasi, narrow nasal bridge, narrow nasal ridge, narrow nasal tip, smooth } \\
\text { philitrum, exaggerated Cupid's bow }\end{array}$ & - \\
\hline P19 & $\begin{array}{l}\text { Motor development delay, small for } \\
\text { gestational age, spasticity, severe learning } \\
\text { disability, epiphyseal changes, short stature }\end{array}$ & $\begin{array}{l}\text { Dolichocephaly, frontal balding, narrow forehead, underdeveloped } \\
\text { supraorbital ridge, full cheeks, malar prominence, premaxillary prominence, } \\
\text { pointed chin, entropion, hypertelorism, sparse eyebrows, almond-shaped } \\
\text { palpebral fissures, telecanthus, angulated antihelix, prominent antihelix } \\
\text { stem, low-set ears, expanded terminal portion crus helix, anterior crease } \\
\text { lobe, thick ala nasi, wide nasal base, depressed nasal bridge, wide nasal ridge, } \\
\text { broad nasal tip, deep philtrum, exaggerated Cupid's bow, thin upper lip } \\
\text { vermilion }\end{array}$ & $\begin{array}{l}\text { Thin corpus } \\
\text { callosum }\end{array}$ \\
\hline P20 & $\begin{array}{l}\text { Neuromotor developmental delay, mild } \\
\text { learning disability, seizure, generalized } \\
\text { hypotonia, progressive vision loss, walking } \\
\text { abnormalities }\end{array}$ & $\begin{array}{l}\text { Broad face, full cheeks, midface prominence, broad jaw, short chin, laterally } \\
\text { extended eyebrows, long eyelashes, telecanthus, angulated antihelix, } \\
\text { prominent antihelix stem, crus helx expanded terminal portion, prominent } \\
\text { crus helix, thick ala nasi, short columella, wide nasal base, wide nasal ridge, } \\
\text { broad nasal tip, smooth philtrum, absent Cupid's bow, thin upper lip } \\
\text { vermilion, wide month }\end{array}$ & $\begin{array}{l}\text { Cortical and } \\
\text { central } \\
\text { atrophy, } \\
\text { enlarged } \\
\text { posterior fossa, } \\
\text { Dandy-Walker } \\
\text { variant }\end{array}$ \\
\hline $\mathrm{P} 21$ & $\begin{array}{l}\text { Neuromotor developmental delay, mild } \\
\text { learning disability, microcephaly, speech } \\
\text { delay, 5th finger clinodactyly }\end{array}$ & $\begin{array}{l}\text { Narrow forehead, metopic depression, depressed glabella, midface } \\
\text { prominence, epicanthus, upslanted palpebral fissure, prominent antihelix } \\
\text { inferior crus, prominent antihelix stem, everted antitragus, low-set ears, wide } \\
\text { nasal base, broad nasal tip, malaligned philtral ridge, thin upper lip vermilion }\end{array}$ & $\begin{array}{l}\text { Dysplastic } \\
\text { corpus } \\
\text { callosum and } \\
\text { polymicrogyria }\end{array}$ \\
\hline $\mathrm{P} 22$ & $\begin{array}{l}\text { Neuromotor developmental delay, mild } \\
\text { learning disability, speech delay, attention } \\
\text { deficit disorder, growth retardation }\end{array}$ & $\begin{array}{l}\text { Full cheeks, malar flattening, midface prominence, epicanthus, telecanthus, } \\
\text { angulated antihelix stem, prominent antihelix stem, low-set ears, prominent } \\
\text { crus helix, thick ala nasi, broad columella, wide nasal bridge, deep philtrum, } \\
\text { exaggerated Cupid's bow }\end{array}$ & Normal \\
\hline
\end{tabular}


Table 2 (continued)

\begin{tabular}{|c|c|c|c|}
\hline Case ID & Presenting symptoms & Dysmorphic facial findings & MRI findings \\
\hline P23 & $\begin{array}{l}\text { Neuromotor developmental delay, } \\
\text { microcephaly, hypotonia in infancy, seizure, } \\
\text { hypospadias, strabismus, nystagmus }\end{array}$ & $\begin{array}{l}\text { Narrow forehead, sloping forehead, full cheeks, midface prominence, short } \\
\text { chin, epicanthus inversus, thick eyebrows, long palpebral fissures, upslanted } \\
\text { palpebral fissures, prominent inferior crus antihelix, prominent antihelix } \\
\text { stem, underdeveloped superior crus antihelix, underdeveloped antitragus, } \\
\text { low-set ears, expanded terminal portion crus helix, small lobes, anteverted } \\
\text { nares, short columella, wide nasal bridge, broad nasal tip, long philtrum, } \\
\text { exaggerated Cupid's bow, wide mouth }\end{array}$ & - \\
\hline $\mathrm{P} 24$ & $\begin{array}{l}\text { Neuromotor developmental delay, } \\
\text { microcephaly, hypotonia, seizure, } \\
\text { strabismus, nystagmus }\end{array}$ & $\begin{array}{l}\text { Brachycephaly, narrow forehead, full cheeks, midface prominence, short } \\
\text { chin, epicanthus inversus, thick eyebrows, long palpebral fissures, prominent } \\
\text { antihelix stem, prominent antihelix crus inferior, underdeveloped antihelix } \\
\text { superior crus, low-set ears, prominent crus helix, expanded terminal portion } \\
\text { crus helix, shell ear, Stahl's ear, anteverted nares, short columella, wide nasal } \\
\text { base, wide nasal bridge, broad nasal tip, long philtrum, tented upper lip } \\
\text { vermilion }\end{array}$ & $\begin{array}{l}\text { Severe cerebral } \\
\text { atrophy, left } \\
\text { frontoparietal } \\
\text { polymicrogy- } \\
\text { ria, bilateral } \\
\text { hyperintense } \\
\text { thalamus }\end{array}$ \\
\hline P25 & $\begin{array}{l}\text { Neuromotor developmental delay, autistic } \\
\text { behaviors, moderate intellectual disability, } \\
\text { language impairment }\end{array}$ & $\begin{array}{l}\text { Brachycephaly, flat occiput, malar flattening, midface prominence, } \\
\text { epicanthus, thick eyebrows, long palpebral fissures, angulated antihelix, } \\
\text { prominent inferior crus of antihelix, prominent antihelix stem, everted } \\
\text { antitragus, underdeveloped crus helix, large lobe, high insertion columella, } \\
\text { narrow nasal base, depressed nasal bridge, convex nasal ridge, narrow nasal } \\
\text { tip, deep philtrum, thick vermilion lower lip, downturned corners of mouth }\end{array}$ & Normal \\
\hline
\end{tabular}

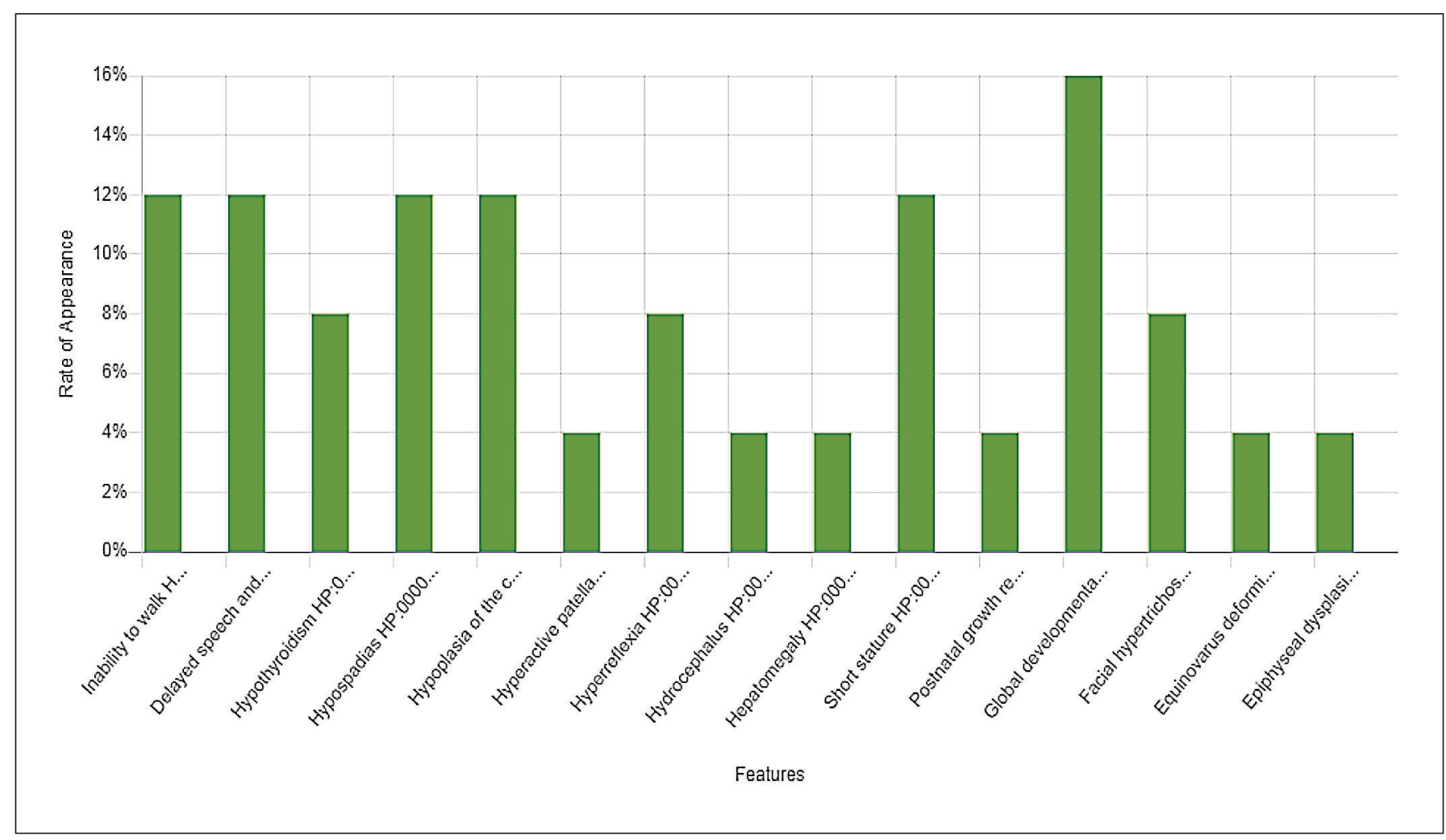

Fig. 1. The distribution of clinical features.

Whole-Exome Sequencing and Artificial Intelligence
Mol Syndromol 2020;11:4-14

DOI: $10.1159 / 000505800$ 
Table 3. Face 2 Gene analysis results

\begin{tabular}{|c|c|c|c|c|c|}
\hline \multirow[t]{2}{*}{$\begin{array}{l}\text { Case } \\
\text { ID }\end{array}$} & \multirow{2}{*}{$\begin{array}{l}\text { Face2Gene } \\
\text { detected } \\
\text { disease }\end{array}$} & \multicolumn{4}{|c|}{$\begin{array}{l}\text { Face } 2 \text { Gene detected same syndrome with WES } \\
\text { results }\end{array}$} \\
\hline & & $\begin{array}{l}\text { rank at } \\
\text { suggested syn- } \\
\text { dromes list }\end{array}$ & $\begin{array}{l}\text { Gestalt } \\
\text { score }\end{array}$ & $\begin{array}{l}\text { Feature } \\
\text { score }\end{array}$ & $\begin{array}{l}\text { Combined } \\
\text { score }\end{array}$ \\
\hline $\mathrm{P} 1$ & + & 2 & 0 & 0.63 & 0.5 \\
\hline P2 & - & & & & \\
\hline P3 & - & & & & \\
\hline P4 & + & 23 & 0 & 0.43 & 0.04 \\
\hline P5 & - & & & & \\
\hline P6 & - & & & & \\
\hline P7 & - & & & & \\
\hline P8 & - & & & & \\
\hline P9 & + & 1 & 0.173054 & 0.38 & 0.51 \\
\hline P10 & - & & & & \\
\hline P11 & - & & & & \\
\hline P12 & - & & & & \\
\hline P13 & + & 4 & 0 & 0.68 & 0.25 \\
\hline P14 & - & & & & \\
\hline P15 & - & & & & \\
\hline P16 & - & & & & \\
\hline P17 & + & 2 & 0 & 0.71 & 0.5 \\
\hline P18 & + & 19 & 0 & 0.51 & 0.06 \\
\hline P19 & - & & & & \\
\hline P20 & + & 21 & 0 & 0.46 & 0.05 \\
\hline $\mathrm{P} 21$ & + & 17 & 0 & 0.49 & 0.07 \\
\hline P22 & + & 15 & 0 & 0.51 & 0.07 \\
\hline P23 & + & 10 & 0 & 0.55 & 0.08 \\
\hline P24 & + & 4 & 0 & 0.59 & 0.25 \\
\hline P25 & + & 12 & 0 & 0.69 & 0.1 \\
\hline
\end{tabular}

diseases, collecting this information on a platform such as Face 2 Gene would be beneficial for the attending physicians. Because these applications are based on deep learning, the more patients analyzed, the higher the accuracy. Especially in rare genetic diseases, it is important to increase the Gestalt score after the analysis of the patient.

Gurovich et al. [2019] reported that the success of Face2Gene top-10 matches was 91\%. In 2019, Mishima et al. reported that Face2Gene success rate was $85.7 \%$ in patients with congenital dysmorphic syndromes in Japan. In the same study, if patients had a diagnosis for which Face2Gene had not been trained, the success of Face2 Gene was $60.0 \%$ [Mishima et al., 2019]. We found low success of Face2Gene in consanguineous marriages. In our study, the recognition rate of Face2Gene application in our patient group was $48 \%$ (This rate was calculated from all diseases in a suggested list of 30 diseases). The probable reason for this low rate may be that the diseases in our patient group are not sufficiently introduced to Face2Gene; the rare genetic diseases are seen uncom- monly in the population, and therefore have only been defined with a few patient photographs in this application.

The Face2Gene application was developed based on facial gestalt analysis. As a result of the face analysis, it determines the Gestalt score for 30 diseases matching the facial features of the patient. Furthermore, this application determines the Feature score for the 30 diseases by analyzing the anamnesis, clinical, laboratory and radiological findings of the patients. We strongly recommend that the clinical findings of the patient should be entered when using Face2Gene application, especially in rare genetic diseases. As seen in Table 3, data of patients with rare genetic diseases are limited in the application. We therefore recommend that physicians register the facial gestalt of patients to Face2Gene during or after diagnosis.

The diagnosis of rare genetic diseases is one of the most challenging fields in clinical genetics. The success rate of WES has been reported to be between 25\% and $57 \%$ in the diagnosis of rare genetic diseases [Boycott et al., 2013; Stark et al., 2016]. However, in this patient group, it may be more helpful to analyze with NGP before, e.g., the DeepLearning program, and then follow traditional diagnostic methods considering the result of NGP. When considering the cost difference between targeted gene panels and WES, with this strategy, a diagnosis with lower costs can be provided [van Nimwegen et al., 2016], and at the same time, we believe the diagnostic procedures can be shortened.

Rare genetic diseases are often inherited in an autosomal recessive manner [Boycott et al., 2013]. Consanguinity between parents is one of the important risk factors for autosomal recessive inherited diseases. In the study of Hamamy et al. [2011], it was reported that the prevalence of congenital anomaly in first-degree cousin marriage offspring increased by $1.7-2.8 \%$ compared to the general population. The rate of consanguineous marriages in Turkey as Muslim and Middle Eastern countries is high. In the study by Kelmemi et al. [2015], the rate of consanguineous marriages in autosomal recessive inherited diseases was reported as $58 \%$ in Tunisia. In our study, 24 of 25 patients had autosomal recessive inherited disease; the rate of consanguineous marriages between the parents of patients with autosomal recessive inheritance was calculated as $80 \%$. This higher rate may be due to the frequent consanguineous marriages in the region. In the study by Kelmemi et al. [2015], the rate of parents sharing the same geographical origin in the autosomal recessive-inherited nonconsanguineous patient group was reported to be $63 \%$.
Elmas/Gogus 
Microcephaly is one of the most common clinical findings seen by medical genetic doctors. Whensearching for "microcephaly" in OMIM, the result was 1,007 from 8,976 entries. On the other hand, von der Hagen et al. [2014] found that $28.5 \%$ of the children with microcephaly had genetic etiology. Also, in a study by Ashwal et al. [2009], 15.5-53.3\% of children with microcephaly had a genetic cause. In our study, the rate of patients with microcephaly who have a genetic disease was $40 \%$. Symptoms such as microcephaly and dysmorphic facial features are common in genetic diseases. Therefore, online databases (such as OMIM) of clinical findings of diseases are helpful tools for clinical genetics. However, medical genetic doctors must decide which of the possible diagnoses is most plausible in the patient. This judgment requires extensive clinical experience.

Evaluating genetic diseases is like working as a detective who investigates a criminal event: every clue is very beneficial for reaching the diagnosis. One of these clues is MRI. Considering that most patients who consulted the genetic clinic are patients with neurological diseases, the importance of MRI is evident [Srivastava et al., 2014]. Corpus callosum abnormality is one of these MR findings. In a review article by Edwards et al. [2014], 30-45\% of the cases with corpus callosum agenesis were identified as genetic causes. In $25-30 \%$, the reason is a single gene mutation. In a study by Schell-Apacik et al. [2008], 32\% of the patients with agenesis of the corpus callosum and dysgenesis of the corpus callosum had a genetic etiology. In our study, corpus callosum abnormality was found in $47 \%$ of the patients with a single gene mutation. The abnormal corpus callosum is a condition that should be taken into consideration by physicians because it may accompany a syndrome.

Nowadays, good anamnesis, examination, radiological and laboratory tests are necessary when diagnosing rare diseases. In addition, recently developed artificial intelligence technology can give us valuable clues in terms of diagnosis. The NGP technology may have a say in the diagnosis of genetic diseases with the use and contribu- tion of people working in this field. So, we recommend employing such NGP programs.

In conclusion, each syndrome has a mask. In other words, all syndromes leave a trace or a clue in the phenotype. For years, dysmorphologists have been looking at photographs in dysmorphology books to diagnose patients' syndromes. With the widespread use of NGP technology in recent years, the diagnosis has become easier, not only by looking, but also by scanning patient pictures. However, introducing clinical findings to the program is crucial for more accurate diagnostic recommendations. Finally, with the expansion of such NGP programs, a large and powerful data library will be created, making future diagnostic procedures easier.

\section{Acknowledgment}

We thank Nicole Fleischer for her support and assistance.

\section{Statement of Ethics}

The project was approved by the University of Afyonkarahisar Health Sciences Ethics Committee, which covered the participating hospitals. All procedures followed were in accordance with the University of Sydney Human Research Ethics Committee and with the Helsinki Declaration of 1975, as revised in 2000 (5). Signed or electronic consent was obtained from all participants in the study.

\section{Disclosure Statement}

The authors have no conflicts of interest to declare.

\section{Author Contributions}

M. Elmas conceived and designed the study. B. Gogus performed clinical assessments, experiments, and contributed to data acquisition, analysis and interpretation. B. Gogus drafted the manuscript. Both authors contributed to critical revision of the manuscript for intellectual content and final approval of the manuscript.
References

Whole-Exome Sequencing and Artificial Intelligence
Allanson JE, Biesecker LG, Carey JC, Hennekam $\mathrm{R}$ : Elements of morphology: introduction. Am J Med Genet A 149A:2-5 (2009).

-Ashwal S, Michelson D, Plawner L, Dobyns WB; Quality Standards Subcommittee of the American Academy of Neurology and the Practice Committee of the Child Neurology Society: Practice parameter: evaluation of the child with microcephaly (an evidence-based review): report of the Quality Standards Subcommittee of the American Academy of Neurology and the Practice Committee of the Child Neurology Society. Neurology 73:887897 (2009).

Baird PA, Anderson TW, Newcombe HB, Lowry RB: Genetic disorders in children and young adults: a population study. Am J Hum Genet 42:677-693 (1988). 
Botstein D, Risch N: Discovering genotypes underlying human phenotypes: past successes for mendelian disease, future approaches for complex disease. Nat Genet 33 Suppl:228-237 (2003).

Boycott KM, Vanstone MR, Bulman DE, MacKenzie AE: Rare-disease genetics in the era of next-generation sequencing: discovery to translation. Nat Rev Genet 14:681-691 (2013).

Clark MM, Stark Z, Farnaes L, Tan TY, White SM, et al: Meta-analysis of the diagnostic and clinical utility of genome and exome sequencing and chromosomal microarray in children with suspected genetic diseases. NPJ Genomic Med 3:16 (2018).

-Edwards TJ, Sherr EH, Barkovich AJ, Richards LJ: Clinical, genetic and imaging findings identify new causes for corpus callosum development syndromes. Brain 137:1579-1613 (2014).

Featherstone K, Latimer J, Atkinson P, Pilz DT, Clarke A: Dysmorphology and the spectacle of the clinic. Sociol Heal Illn 27:551-574 (2005).

Ferry Q, Steinberg J, Webber C, FitzPatrick DR, Ponting CP, et al: Diagnostically relevant facial gestalt information from ordinary photos. Elife 3:e02020 (2014).

-Guest SS, Evans CD, Winter RM: The Online London Dysmorphology Database. Genet Med 1:207-212 (1999).

Gurovich Y, Hanani Y, Bar O, Fleischer N, Gelbman D, et al: DeepGestalt - identifying rare genetic syndromes using deep learning. arXiv:1801.07637 (2018).
Gurovich Y, Hanani Y, Bar O, Nadav G, Fleischer $\mathrm{N}$, et al: Identifying facial phenotypes of genetic disorders using deep learning. Nat Med 25:60-64 (2019).

Hamamy H, Antonarakis SE, Cavalli-Sforza LL, Temtamy S, Romeo G, et al: Consanguineous marriages, pearls and perils: Geneva International Consanguinity Workshop Report. Genet Med 13:841-847 (2011).

Hammond P: The use of 3D face shape modelling in dysmorphology. Arch Dis Child 11201126 (2007).

Kelmemi W, Chelly I, Kharrat M, ChaabouniBouhamed H: Consanguinity and homozygosity among Tunisian patients with an autosomal recessive disorder. J Biosoc Sci 47:718726 (2015).

Lodato E, Kaplan W: Priority Medicines for Europe and the World: 2013 Update Report, chapter 6.1, pp 68-74 (WHO 2013).

Mishima H, Suzuki H, Doi M, Miyazaki M, Watanabe S: Evaluation of Face2Gene using facial images of patients with congenital dysmorphic syndromes recruited in Japan. J Hum Genet 64:789-794 (2019).

Ng SB, Turner EH, Robertson PD, Flygare SD, Abigail W, et al: Targeted capture and massively parallel sequencing of 12 human exomes. Nature 461:272-276 (2009).

Ng SB, Nickerson DA, Bamshad MJ, Shendure J: Massively parallel sequencing and rare disease. Hum Mol Genet 19:119-124 (2010).

Pantel JT, Zhao M, Mensah MA, Hajjir N, Hsieh TC, et al: Advances in computer-assisted syndrome recognition by the example of inborn errors of metabolism. J Inherit Metab Dis 41: 533-539 (2018).
Schell-Apacik CC, Wagner K, Bihler M, ErtlWagner B, Heinrich U, et al: Agenesis and dysgenesis of the corpus callosum: clinical, genetic and neuroimaging findings in a series of 41 patients. Am J Med Genet A 146A:25012511 (2008)

Smigiel R, Demkow U: Next generation sequencing in dysmorphology, in Demkow U, Ploski R (eds): Clinical Applications for Next-Generation Sequencing (Elsevier Inc., London 2016).

Srivastava S, Cohen JS, Vernon H, Barañano K, McClellan R, et al: Clinical whole exome sequencing in child neurology practice. Ann Neurol 76:473-483 (2014).

Stark Z, Tan TY, Chong B, Brett GR, Yap P, et al: 2016. Original Research Article A prospective evaluation of whole-exome sequencing as a first-tier molecular test in infants with suspected monogenic disorders. Genet Med 18: 1090-1096 (2016).

Strømme P: The diagnosis of syndromes by use of a dysmorphology database. Acta Paediatr Scand 80:106-109 (1999).

van Nimwegen KJ, van Soest RA, Veltman JA, Nelen MR, van der Wilt GJ, et al: Is the $\$ 1000$ Genome as near as we think? A cost analysis of next-generation sequencing. Clin Chem 62:1458-1464 (2016).

$\checkmark$ von der Hagen M, Pivarcsi M, Liebe J, von Bernuth $\mathrm{H}$, Didonato $\mathrm{N}$, et al: Diagnostic approach to microcephaly in childhood : a twocenter study and review of the literature. Dev Med Child Neurol 56:732-741 (2014).

Yang Y, Muzny DM, Xia F, Niu Z, Person R, et al: Molecular findings among patients referred for clinical whole-exome sequencing. JAMA 312:1870-1879 (2014). 